\title{
Lange Zeit ein Tabuthema
}

\section{Jean Martin}

Dr. med., Mitglied der Redaktion

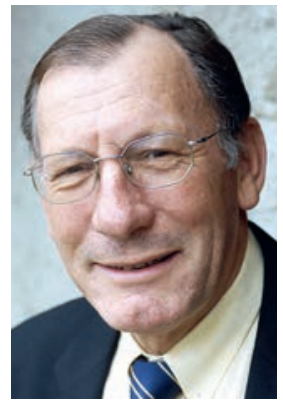

Zwei Hochschuldozenten veröffentlichten jüngst ein grosses Werk zum Thema Coaching, vor allem im Klinikbereich. Das Thema stand in der Vergangenheit kaum im Mittelpunkt des Interesses, aber wir alle erleben (oder erlebten) Situationen, in denen seine Bedeutung offenkundig wird. Den Mitarbeitenden im Gesundheitswesen, Ärzten und anderen Betroffenen, sind die Schwierigkeiten im zwischenmenschlichen Bereich, die Hybris einiger Vorgesetzter, unzureichende Kompetenzen in Sachen Personalführung oder Management, die Antagonismen und Eifersüchteleien wohl bekannt. Wie oft wurden Sitzungen renommierter Kommissionen oder Fakultätsräte als laut und konfus agierender Kindergarten beschrieben ... Jüngst eingesetzte Chefärzte/Chefärztinnen stellen - beispielsweise mit Blick auf allfällig vorhandene Rivalen/Rivalinnen - nur gering entwickelte Psychologiekenntnisse unter Beweis (oder aber auch gute Beziehungsfähigkeit). Es gibt auch Fälle, in denen der Berufskollege Prügelknabe des Chefs oder des ganzen Teams ist.

All dies blieb lange Zeit im Unausgesprochenen oder Informellen, unscharf, heikel. Viele von uns suchten solche leidvollen oder gar gewaltgeprägten Situationen zu lindern bzw. aufzulösen. «Bestmöglich», mit Hilfe der jeweiligen Kompetenzen und des jeweiligen Charismas - mit unterschiedlichem Erfolg ...

Die Autoren von «Coaching des soignants» wurden nach erfolgreicher Berufslaufbahn - dazu geführt, in ebensolchen Situationen Mandate anzunehmen und sich in der Folge weiterzubilden, wieder die «Schulbank» zu drücken. Nach vierzig Jahren in der Reanimation/Intensivpflege wurde Prof. René Chioléro vom CHUV so zum zertifizierten Coach, desgleichen Véronique Haynal, Psychologin und Rehabilitationstherapeutin in Genf.

Nach einer Einführung in die Philosophie des Coaching teilt sich das Werk in zwei grosse Teile. Der erste Teil (Les fondamentaux, "die Grundlagen») befasst sich mit den Bereichen Coaching und Mentoring (nicht dasselbe!), mit der Bedeutung von Supervision und dem Bezug zur Psychotherapie. Es folgt La pratique du coaching hospitalier («die Praxis des Coaching im Klinikbereich»). Kapitel 5 behandelt beispielsweise die Pflegenden und ihre Berufskultur. Wir sind uns dessen nicht immer bewusst. Dazu gehört sehr viel, die Bedeutung und die Konsequenzen dieser Kultur, die Sozialisierung und Formatierung, die wir erlebt und/oder von denen wir profitiert haben. Kapitel 12 erörtert den Burnout und seine Prävention (sich zu pflegen, Erlernen von «Achtsamkeit»), Kapitel 13 ist mit Konflikten und Gewalt befasst. Zum Schluss beschreiben die Autoren ihre eigenen Lebenswege und zeigen Perspektiven auf.

Mehr Coaching im Gesundheitssystem ist wünschenswert. Dazu braucht es eine andere Einstellung - auch mehr «Herz» - wie in anderen Entwicklungen auch. Als es beispielsweise üblich wurde, dass Patienten eine zweite ärztliche Meinung einzuholen begannen, war dies vom Arzt nicht gerne gesehen, es wurde als Vertrauensmangel aufgefasst. Falsch! Das Einholen einer zweiten Meinung ist ein verständlicher Wunsch, der vom behandelnden Arzt einfach als solcher zu verstehen ist. Zugang zur Patientenakte: Einige sträubten sich lange dagegen, dass dem Patienten aus rechtlicher und ethischer Sicht vollkommene und unverzügliche Einsicht zu gewähren ist. So sollte auch das Coaching in schwierigen Situationen von allen begrüsst werden, auch von Kollegen, die sich für wichtig halten; dies vor allem angesichts des Potenzials, das Coaching besser als zuvor zur Entlastung und idealerweise zur Regelung von Problemsituationen, in die wir ja nur allzu häufig geraten, bietet.

"Coaching des soignants" ist eine praktische Abhandlung, bestückt mit Abbildungen und vielen Skizzen aus dem praktischen Alltag - R. Chioléro führt u.a. einige Beispiele an, in die er selbst involviert war. In all diesen Fällen wird die Ausgangssituation beschrieben, die Kontakte zum Coach, das Mandat und die verschiedenen Etappen. Allerdings wird das Ergebnis - ausser in wenigen Fällen - nicht präzisiert («happy end», schwierige Thematik, Misserfolg?). Das hat mich beschäftigt. Vielleicht weil das Augenmerk auf den Prozess gesetzt wird?*

Das Werk ist kein page-turner im angelsächsischen Sinne, doch es ist in jedem Fall wert, sich hinein zu vertiefen. Es leistet einen wichtigen Beitrag zum besseren Verständnis und zu einer besseren Umsetzung von Coaching in der Pflege.

\footnotetext{
In Zeiten von Me-too, in denen auch aufgezeigt wird, wie häufig unangemessene, sexuell konnotierte Gesten auch im medizinisch klinischen Bereich vorkommen (dieser Bereich ist ganz sicher keine Ausnahme), wird insgesamt nur einmal der Begriff "sexistische Bemerkungen» (S. 342) angeführt und einmal der Begriff "Sexismus» (S. 382). Erstaunlich, nicht wahr? Auf meine diesbezügliche Frage zeigte sich auch der erste Autor verblüfft.
} 